\title{
DEFINICIÓN DE ESTÁNDARES EN COMPETENCIAS INFORMACIONALES EN COMUNICACIÓN CIENTÍFICA Y SU APLICACIÓN EN DOCENTES UNIVERSITARIOS MEXICANOS
}

\author{
Rocío Anchondo-Granados* \\ Universidad Autónoma de Chihuahua (México) \\ Javier Tarango Ortiz ** \\ Universidad Autónoma de Chihuahua (México) \\ Jesús Cortés-Vera ${ }^{* * *}$ \\ Universidad Autónoma de Chihuahua (México) \\ Juan Daniel Machin Mastromatteo ${ }^{* * *}$ \\ Universidad Autónoma de Chihuahua (México)
}

\begin{abstract}
Resumen: La presente investigación expone un conjunto de estándares compuestos de distintas dimensiones e indicadores de rendimiento para evaluar la competencia en la comunicación científica en profesores universitarios mexicanos. El estudio se integró por distintas fases complementarias y subsecuentes: (i) investigación documental sobre elementos normativos y teóricos que sustentan la identificación inicial de indicadores de rendimiento de evaluación comunicación científica; (ii) validación de información por 32 investigadores expertos en ciencias; (iii) validación de información por 62 expertos en alfabetización informacional (ALFIN); y (iv) derivación de un conjunto de estándares, mismos que fueron probados en 28 profesores universitarios del área de las ciencias químicas con potencialidad científica. Los resultados de la investigación definieron un conjunto de estándares integrados por ocho dimensiones y 34 indicadores de rendimiento, para posteriormente probar su funcionalidad diagnosticando niveles individuales y colectivos de competencia en comunicación científica, favoreciendo la identificación de fortalezas y debilidades, con lo cual se posibilita el diseño de propuestas de mejora a través de procesos planeados de cambio y beneficiando el desarrollo de habilidades hacia la producción y comunicación científica.
\end{abstract}

Palabras clave: comunicación científica; alfabetización científica; alfabetización informacional; cultura científica; estándares de competencia científica.

Title: DEFINITION OF STANDARDS IN INFORMATION COMPETENCES IN SCIENTIFIC COMMUNICATION AND THEIR APPLICATION IN MEXICAN UNIVERSITY TEACHERS.

Abstract: The present research exposes a set of standards composed of different dimensions and performance indicators to evaluate the competence in scientific communication in Mexican university professors. The study consisted of different complementary and subsequent phases: (i) documentary research on normative and theoretical elements that support the initial identification of performance indicators for scientific communication evaluation; (ii) validation of information by 32 expert researchers in science; (iii) validation of information by 62 experts in information literacy (IL); and (iv) derivation of a set of standards, which were tested by 28 university professors in the area of chemical sciences with scientific potential. The results of the research defined a set of standards made up of eight dimensions and 34 performance indicators, to later test their functionality by diagnosing individual and collective levels of competence in scientific communication, favoring the identification of strengths and weaknesses, thereby enabling the design of improvement proposals through planned processes of change and benefiting the development of skills towards scientific production and communication.

Keywords: scientific communication; scientific literacy; information literacy; scientific culture; standards of scientific competence.

Copyright: (C) 2020 Servicio de Publicaciones de la Universidad de Murcia (Spain). Este es un artículo de acceso abierto distribuido bajo los términos de la licencia Creative Commons Reconocimiento 4.0 Internacional (CC BY 4.0).

\footnotetext{
* roxioag@gmail.com

*** tj.88888@hotmail.com

*** jcortes@uacj.mx

**** jmachin@uach.mx
}

Recibido: 21-05-2019; 2a versión: 12-04-2020; aceptado: 14-04-2020.

ANCHONDO GRANADOS, R.; TARANGO ORTIZ, J.; CORTÉS VERA, J. y MACHIN MASTROMATTEO, J.D. Definición de estándares en competencias informacionales en comunicación científica y su aplicación en docentes universitarios mexicanos. Anales de Documentación, 2020, vol. 23, n² 2. Disponible en: http://dx.doi.org/10.6018/analesdoc.379381. 


\section{INTRODUCCIÓN}

Para medir la competencia científica, es necesaria la sólida presencia de niveles adecuados de ALFIN y de comunicación científica, de lo cual surge la necesidad de identificar estándares para su medición de forma concreta. La apreciación de los niveles de ALFIN sucede a través de la identificación y desarrollo de habilidades para buscar, evaluar y utilizar información; en cambio, para la comunicación de la ciencia, se pretende encontrar formas de construcción de conocimiento, a través de publicaciones de calidad. Incluso podría decirse que la comunicación científica es consecuencia de la ALFIN, siendo su resultado óptimo, la demostración de la competencia científica en donde se conjugan ambos conceptos.

Tanto ALFIN, como la comunicación de la ciencia, deben relacionarse con procesos educativos, ya que su objetivo es procurar competencias para un correcto aprendizaje y desarrollo de destrezas en los nuevos entornos (Tarango y Mendoza Guillén, 2012). Así también, existen formas específicas de trabajo en el proceso de enseñanza-aprendizaje, el cual busca que un individuo o un colectivo alcancen las competencias (conocimientos, habilidades y actitudes) en lo informático, comunicacional e informacional. Para ello, se recurre a utilizar diferentes formatos, medios y recursos físicos, electrónicos o digitales en forma adecuada y eficiente, con una posición crítica y ética, a partir de sus potencialidades (cognoscitivas, prácticas y afectivas) y conocimientos previos (otras alfabetizaciones) (Tójar y Serrano, 2012; Uribe Tirado, 2009).

Los procesos de comunicación de la ciencia comienzan a consolidarse como un ámbito creciente, con la formación de indicadores de rendimiento institucionales para su buen ejercicio, este quehacer requiere de más apoyos sólidos y congruentes. Además, nace la necesidad de incorporar una cultura científica, en forma generalizada, entre la población académica, desarrollar líneas de acción que protejan la labor del comunicador de la ciencia. En otras palabras, se debe en cierta manera, formalizar estándares en los cuales los investigadores puedan apoyarse para cumplir sus funciones.

La generación de conocimiento cobra importancia a partir de que el objetivo primario de la comunicación científica es favorecer la diseminación y el intercambio de los resultados de la investigación, con vistas a acelerar el desarrollo científico, tecnológico, económico y social de la humanidad. Finalmente, cuando un autor se propone desarrollar alguna publicación científica, su aspiración es que esta alcance la mayor audiencia posible, buscando con su propia competencia diversas formas para obtener una mayor visibilidad, tanto multidisciplinaria como especializada (Cañedo Andalia, 2013).

De forma reciente, las políticas nacionales en ciencia y tecnología de México, han desarrollado iniciativas que promueven la comunicación científica por parte de profesores universitarios, cuya participación ha propiciado el crecimiento de sus condiciones de generación de conocimiento y de reducción de la brecha de dependencia cognitiva en relación con países altamente desarrollados, no obstante, muchos de ellos no están lo suficientemente preparados para migrar de su condición de ágrafos científicos. Este artículo es una derivación de los resultados globales obtenidos a través de la tesis de doctorado en educación de Anchondo-Granados (2017), titulada "Evaluación de competencias en comunicación científica: definición de un conjunto de estándares para docentes universitarios”, a través de la cual se proponen herramientas diagnósticas hacia la búsqueda de la mejora continua.

\section{ALFABETIZACIÓN CIENTÍFICA COMO CONDICIÓN DE LA COMPETENCIA EN COMUNICACIÓN CIENTÍFICA}

La sociedad está prestando una atención creciente a los datos y la información, particularmente en dominios generales y científicos, se refleja en la iniciativa de los datos abiertos y en la contribución de la ALFIN, como parte de la misión de fomentar el conocimiento y la innovación. Calzada Pardo y Marzal (2013), consideran necesario hablar de una combinación de elementos entre ALFIN y ciencia, lo que dará como resultado la llamada alfabetización científica, cuyo éxito sucede a partir del desarrollo de habilidades en esta competencia, generando docentes capacitados para tomar decisiones asertivas en cuanto al saber y hacer científico.

La concepción de la alfabetización científica supone múltiples características definitorias: (i) la capacidad de leer y escribir sobre ciencia y tecnología (Teixidó Mans, 2012); (ii) la comprensión pública de la ciencia y la tecnología debe diferenciar entre alfabetización científica práctica, alfabetización científica cultural y alfabetización científica cívica (González Rosario y Hernández Lárez, 2009); (iii) inicia a partir de un esfuerzo por comprometer a los científicos en la tarea comunicativa, con el objetivo de mejorar la capacidad de entender los fenómenos (García Gómez y Martínez Bernat, 2010); y (iv) tiende a fomentar un pensamiento crítico y a desarrollar competencias de acción para la toma de decisiones, en donde el tratamiento global y multidimensional exige la interpretación de los problemas sociales y por 
consecuencia, se traduce igualmente en la exigencia de una educación integral (Martín Gutiérrez y Tyner-Austin, 2012).

La competencia científica comprende un conjunto de conocimientos que poseen los investigadores, así como destrezas y actitudes para interpretar, usar y apropiar los productos de la ciencia y la tecnología, cuando se toman decisiones de carácter tecno-científico o sobre sus productos y sus modos de consumo (Torres Mesías, Mora Guerrero, Garzón Velázquez y Ceballos Borina, 2013). Además, poseer un bagaje suficiente de conocimientos sobre hechos, conceptos, estructuras cognitivas y habilidades, discutir sobre temas científicos actuales y documentarse constantemente (López Blanco, 2004).

La labor de la alfabetización científica y el trabajo epistemológico basado en la comprensión de la naturaleza han demostrado ser herramientas de gran utilidad para favorecer los procesos constructivos de la generación de conocimiento (Ortega, 2011). En tiempos actuales, el análisis de la actividad científica en instituciones académicas de nivel superior es una tarea fundamental para conocer su eficacia y eficiencia en el uso de los recursos destinados al desarrollo (Peralta González, Solís Cabrera y Peralta Suárez, 2011). La mayoría de las mediciones suceden mediante la aplicación de técnicas métricas, pero también debe considerarse la medición individual personal, de capacidades reales y de percepción personal hacia la ciencia y la construcción de conocimiento (De Pablos Pons, 2010). Incluso, es posible romper las barreras limitadoras de las disciplinas curriculares, si se generan nuevas formas de aprender con una visión abierta e interdisciplinar.

La didáctica actual de las ciencias promueve la alfabetización científica como finalidad central de su enseñanza, ya que se basa en los siguientes aspectos fundamentales: (i) identificar los propósitos de la enseñanza de las ciencias; (ii) generar mecanismos para el conocimiento de la naturaleza de la ciencia y de las relaciones ciencia-tecnología y sociedad; (iii) promover la identificación de temas de la ciencia de frontera, aplicados a tomar decisiones en la vida personal o de la sociedad; (iv) generar mecanismos procedimentales para la obtención y uso de la información científica; y (v) desarrollar capacidades afectivas (emociones, actitudes, valores y disposición) ante la alfabetización científica; esto es, incrementar el aprecio e interés por la ciencia (Martín Díaz, 2005; Villarruel-Fuentes, 2013; Gavidia, 2013).

El docente universitario, tanto como destinatario y productor del discurso académico-científico, está llamado a buscar su propio desarrollo científico y el de sus estudiantes como futuros investigadores, centrándose principalmente en la lectura crítica de contenidos y en la consiguiente construcción de escritos. Este andamiaje es necesario en toda comunidad académica que promueva los retos intelectuales para desarrollar de manera autónoma las destrezas lingüísticas, conceptuales y metodológicas. La cultura científica debiera ser una responsabilidad compartida del docente, del estudiante y de la institución (Torres Mesías, Mora Guerrero, Garzón Velázquez y Ceballos Borina, 2013). Desafortunadamente, la mayoría de las experiencias de enseñanza de la lectura y escritura académica y científica en Latinoamérica son iniciativas individuales y aisladas, que en muy pocos casos involucran el diseño de políticas institucionales para que los profesores se formen como seres críticos capaces de interpretar y producir textos de manera autónoma, fortaleciendo así las competencias comunicativas e investigativas (Larraín Sutil, 2009; Cárdenas Casique, 2013).

La alfabetización científica del siglo XXI no puede limitarse únicamente a aspectos conceptuales o procedimentales, debe además comprender competencias relacionadas con el intercambio dialógico, la participación, la evaluación y la confrontación de intereses y valores presentes en las decisiones relacionadas en el desarrollo tecnocientífico (Caamaño, 2011; Sanz y López, 2012). Lo anterior lleva a la necesidad de adquirir competencias adicionales, específicamente aquellas vinculadas con aspectos instrumentales, de conocimientos y de valores (Area Moreira, 2008). Por tanto, la cultura científica va más allá de la mera transmisión de resultados y avances, teniendo más que ver con el desarrollo humano y social: el análisis, la creatividad, la crítica constructiva, el trabajo colectivo, la síntesis, la adaptación a los cambios, la evaluación, la capacidad para afrontar las consecuencias de nuestros pensamientos y acciones, la comunicación para el enriquecimiento cultural, la generación de valor agregado al conocimiento y el interés permanente, además de la conciencia intercultural (Wilson, Grizzle, Tuazon, Akyempong y Cheung, 2011; Martín y Tyner 2012; Fong, 2013).

Tanto la ALFIN como la alfabetización científica y la cultura científica en su conjunto, tienden a ser medidas a través de productos de investigación científica (Izquierdo, 2013), demandando la necesaria recurrencia al uso de la información científica cuyo resultado se proyecte en nuevos planteamientos, discusiones públicas relacionadas con la ciencia y la tecnología, además de la realización personal del docente como investigador (Colorado Zúñiga y Rodríguez Mosquera, 2014). Todo esto ocasiona la necesidad de considerar múltiples variables relacionadas con habilidades y conocimientos culturales (Harlen, 2002; Valdés Cuervo, Vera Noriega y Carlos Martínez, 2012): (i) 
identificación de cuestiones científicas mostrando aptitud y astucia para entender los hechos científicos; (ii) describir, explicar y predecir fenómenos científicos y sociales; (iii) predecir cambios en la evolución de la ciencia; (iv) identificar las descripciones, explicaciones y pronósticos apropiados; (v) utilizar pruebas científicas, así como planear y evaluar argumentos científicos y tecnológicos basados en evidencias; (vi) lectura comprensiva que diferencie entre comunicación científica y divulgación científica; y (vii) capacidad para evaluar la calidad de la información científica.

La importancia de la comunicación científica como producto clave de este estudio, se fundamenta en las siguientes consideraciones: (i) el papel de las ciencias en la educación va más allá de una necesidad formativa solo de estudiantes; (ii) la ciencia está estructurada por un conjunto de conceptos, leyes y teorías utilizadas para interpretar fenómenos, pero también por los procedimientos para generarlos, validarlos y modificarlos; (iii) la competencia científica es determinante para el desarrollo económico de un país; y (iv) el alfabetismo científico va más allá de tener conocimientos sobre ciencia, considera también la generación de nuevos conocimientos (Jiménez y Otero, 2012). La investigación científica ha tenido que cambiar los paradigmas que implican el mero adiestramiento conceptual y teórico, por aquellos que consideran las dimensiones sociales relacionadas a una cultura científica (Gavidia, 2013). Además, la alfabetización científica propone objetivos básicos para todos los docentes, evitando desigualdades sociales en educación (Hernández, Flavia y Zacconi, 2010; De Andrea González y Tena Godoy, 2012).

\section{DISEÑO DE LA INVESTIGACIÓN}

\subsection{Propósitos de la investigación}

El propósito de esta investigación fue desarrollar un conjunto de estándares para identificar los niveles de alfabetización científica en relación con las prácticas de comunicación científica, cuyo resultado permita fortalecer en un mejor desarrollo del conocimiento científico. Además, se buscó estudiar la calidad con respecto a la comunicación (escrita y verbal) en razón de las fortalezas y debilidades de los investigadores universitarios mexicanos.

\subsection{Metodología del estudio}

Esta investigación se caracterizó por los siguientes aspectos metodológicos:

- La recolección de datos iniciales se basó en los procesos de investigación documental, siendo necesario el acceso a diversas fuentes que, de forma directa o indirecta, se refirieran a la definición de competencias en comunicación científica.

- Se utilizó un enfoque mixto de investigación en dos etapas: (i) de carácter cualitativo, en la integración de los indicadores de rendimiento a incluir en los estándares a través de entrevistas a profundidad y usando medios electrónicos para validación de datos; y (ii) de carácter cuantitativo, en su aplicación para diagnóstico de condiciones de investigadores potenciales.

- Por tanto, el proyecto estuvo compuesto por cuatro fases: (i) Fase 1. Investigación documental a través de los indicadores de rendimiento de diferentes instituciones respecto a la medición de la comunicación científica; (ii) Fase 2. Validación de los resultados a través de investigadores universitarios de áreas de las ciencias químicas y áreas afines para la identificación de indicadores de rendimiento; (iii) Fase 3. De acuerdo a los resultados anteriores, validación de los resultados con expertos en ALFIN; y (iv) Fase 4. Aplicación de los estándares en docentes universitarios con potencialidad científica.

\section{PRESENTACIÓN Y ANÁLISIS DE RESULTADOS}

En el caso de México, no existen normas específicas que identifiquen y diferencien niveles de comunicación científica (individual o colectiva), por lo que el estudio tomó como punto de partida diversas visiones institucionales particulares y propuestas nacionales sobre la perspectiva existente del comportamiento productivo de investigadores.

\subsection{Fase 1. Generación de la propuesta inicial}

Al no existir normatividad relacionada con la evaluación de competencias en comunicación científica, se consideró pertinente tener como punto de partida diversos lineamientos de convocatorias vinculadas a la producción científica, entre ellas: (i) criterios internos de evaluación por área de conocimiento del Sistema Nacional de Investigadores (SNI) del Consejo Nacional de Ciencia y Tecnología (CONACYT, 2019a), por nivel y por área de conocimiento (CONACYT, 2019b); (ii) criterios de evaluación del Programa de Mejoramiento del Profesorado (Secretaría de Educación Pública [SEP], 2019a); (iii) criterios de evaluación de Cuerpos Académicos (SEP, 2019b); (iv) lineamientos del programa de carrera docente en universidades públicas estatales (SEP, 2018); (v) criterios de evaluación de investigadores de centros de investigación públicos (CONACYT, 2019c); (vi) criterios de evaluación institucional de observatorios científicos nacionales (Universidad Nacional Autónoma de México [UNAM], 2019); 
(vii) documentos base para regular la ciencia en México (CONACYT, 2014d); y (viii) revisión de la literatura científica pertinente (Aguilar Justo, Gallegos Ramírez y Medina Ramírez, 2013; Izquierdo, 2013; Palomares Ruiz, Sordia Salinas y Dimas Rangel, 2015; Buela-Casal, Quevedo Blasco y Guillén Riquelme, 2015; Buela Casal et al., 2015; Tarango y Machin-Mastromatteo, 2017).

El resumen resultante del análisis documental anterior permitió identificar 47 indicadores de rendimiento iniciales, mismos que se compactaron en esta fase en 24, bajo la siguiente propuesta: (i) aspectos relacionados con la escritura científica, a través de publicaciones: artículos (indizados, arbitrados y de divulgación), libros, capítulos de libro y memorias en extenso; (ii) actividades vinculadas con el ámbito académico y la formación de capital humano: dirección de tesis y desarrollo de materiales didácticos; (iii) aspectos relacionados con la innovación: desarrollo de patentes, proyectos de investigación y desarrollo de modelos y prototipos; (iv) interrelación con otros ámbitos de la ciencia: citas, relación con otros miembros de diversas comunidades científicas, ofrecimiento de asesorías y consultorías; y (v) calidad de los elementos académicos y científicos: nivel de obsolescencia de las publicaciones, grados académicos, dominio de otros idiomas, recepción de premios y reconocimientos nacionales o internacionales como investigador y otorgados por comunidades científicas o de evaluación y resultados de evaluación relacionados con la calidad docente y participación en programas académicos con acreditación por su calidad.

\subsection{Fase 2. Validación de indicadores de rendimiento de propuesta inicial por investigadores del área científica}

A partir del listado inicial de indicadores de rendimiento de identificación de las funciones de comunicación de la ciencia, se procedió a validar dicho listado a través de un grupo de expertos de las ciencias química y áreas afines, como biología, medicina y bioquímica; esto debido a que los criterios de evaluación del SNI-CONACYT para las áreas mencionadas son los de mayor exigencia. Se tomaron como referencia para la selección de estos expertos a instituciones de educación superior del Estado de Chihuahua, México, tales como: (i) Centro de Investigación en Materiales Avanzados (CIMAV); (ii) Centro de Investigación en Alimentos y Desarrollo (CIAD); (iii) Universidad Autónoma de Ciudad Juárez (UACJ), Instituto de Ciencias Biomédicas (ICB); y (iv) Universidad Autónoma de Chihuahua (UACH), facultades de Medicina y Ciencias Químicas.

Para esta fase, la selección de la muestra fue por conveniencia y se seleccionó a los participantes con las siguientes características: (i) Grado de doctor en ciencias; (ii) pertenencia a los niveles II o III del SNI; (iii) laborar de tiempo completo en las instituciones seleccionadas; y (iv) pertenencia a las áreas disciplinares de interés al estudio. Así, se recolectaron datos de 26 investigadores de un total de 32 que se identificaron que cumplían los criterios de selección mencionados. Se realizaron entrevistas personales con los 32 investigadores para explicar los propósitos del estudio y se les presentó el listado original de criterios, para obtener su validación, a través de las siguientes opciones: (i) selección de la inclusión o exclusión del criterio; (ii) proposición de otros criterios a considerar en el listado; (iii) emisión de observaciones y justificaciones de inclusión o exclusión; y (iv) priorización de elementos de acuerdo a su nivel de importancia.

Como parte del proceso de validación, además de la priorización de criterios se recolectaron diversas recomendaciones respecto a esta clase de procesos, entre las que destacan: (i) la necesidad de estandarizar, más que normalizar, los lineamientos que se establezcan para el reconocimiento de las funciones del investigador; y (ii) establecer elementos a través de los cuales el investigador conozca su trabajo y cuente con un referente mínimo, para no verse limitado en suficiencia de comunicación científica, ni abrumado por exceso de productos.

Para iniciar la integración de elementos de fondo y forma de los estándares de comunicación científica, se ordenaron los 34 indicadores de rendimiento aceptados y se decidió su agrupación en ocho dimensiones: (i) generación de conocimiento; (ii) aplicación de la innovación; (iii) impacto de la producción científica; (iv) calidad académica y habilitación profesional; (v) ámbitos para el ejercicio profesional; (vi) procesos de investigación; (vii) aspectos éticos y legales; (viii) transferencia de conocimiento. A este proceso de validación desarrollado por investigadores siguió como procedimiento principal solicitar al participante evaluar cada criterio, asignando una ponderación de uno (1) cuando se considera prioritario y cero (0) al no considerarlo, además de complementar su calificación con comentarios que la justificaran.

Los indicadores de rendimiento que mostraron resultados más relevantes, incluso a nivel de unanimidad, son los que reconocen a la competencia en comunicación científica, aquellos en los que el investigador logra demostrar: (i) conocimientos suficientes sobre redacción científica; (ii) manejo adecuado de referencias y citas de información científica; (iii) apego a manuales de estilo, especialmente los que demandan las revistas científicas; (iv) acceso a bases de datos y uso de estrategias de búsqueda de información; (v) análisis y síntesis de información; (vi) identificación de medios de comunicación científica pertinentes y uso de la literatura científica; y (vii) diferenciación entre los medios 
de comunicación científica y los de divulgación científica. Los criterios de ponderaciones inferiores fueron eliminados, con lo cual se generó la segunda versión de los estándares como parte final de la fase 2 de la investigación.

Los criterios que los investigadores validaron para su eliminación fueron: (i) tener patentes, tanto aprobadas como en proceso de aprobación; (ii) recibir premios de reconocido prestigio nacional e internacional sobre la capacidad de desarrollar producción científica; (iii) poseer algún reconocimiento académico de acreditación docente dentro de la institución de trabajo; (iv) ofrecer servicios profesionales de asesoría o consultorías profesionales o académicas en entidades distintas a las de su ámbito laboral; y (v) ser instructor de cursos y talleres.

\subsection{Fase 3. Validación de los indicadores de rendimiento por expertos en ALFIN}

En esta fase se consultó la base de datos de acceso abierto "Directorio de Expertos en el Tratamiento de la Información” conocida como EXIT (http://www.directorioexit.info), donde se identificaron aproximadamente 300 expertos internacionales, cuya línea disciplinar de trabajo fuera la ALFIN o alguna vertiente adicional, como: alfabetización digital, habilidades informacionales y competencias informacionales, a quienes por medio de un muestreo por conveniencia, se les envió un correo masivo para solicitar su validación de los criterios de evaluación, lográndose 62 participaciones provenientes de: Alemania, Argentina, Austria, Brasil, Chile, Colombia, Cuba, Ecuador, Egipto, España, Estados Unidos, Francia, India, Italia, Marruecos, México, Perú, Portugal, Reino Unido, Rumania, Uruguay y Venezuela.

La validación realizada por expertos en ALFIN consistió principalmente en solicitarles que indicaran: (i) priorización de dimensiones; y (ii) evaluación de indicadores de rendimiento, con la posibilidad de agregar comentarios para su mejoramiento, observándose solo la recomendación de fusionar dos indicadores de rendimiento: ‘conocer y aplicar las normas de derechos de autor de tal forma que no recae en el plagio o autoplagio' y 'conocer y aplicar las normas de propiedad intelectual vigentes, enfocadas principalmente al desarrollo tecnológico' por 'conocer y aplicar las normas de derechos de autor y de propiedad intelectual sin recaer en el plagio y autoplagio’.

A partir de los procesos de análisis y recolección de datos antes descritos, fue posible conjuntar los resultados de las validaciones de las fases 2 y 3, reduciendo la lista de indicadores de rendimiento propuestos de 41 a 34, los cuales se categorizaron en ocho dimensiones. La estructura resultante conformó el conjunto de estándares titulado: ‘Estándares sobre aptitudes-facultades en comunicación científica: indicadores de rendimiento’ (ver Anexo 1).

\subsection{Fase 4. Aplicación del estándar a docentes con potencialidad científica}

Posterior a la validación del instrumento y la integración del conjunto de estándares, se procedió a aplicarlos en docentes identificados con potencialidad científica y pertenecientes a las instituciones participantes antes mencionadas, tomando como referencia los siguientes indicadores de rendimiento: (i) pertenecer a la disciplina científica de la química y áreas afines como biología, medicina y bioquímica; (ii) tener grado de doctorado; (iii) mostrar aspiración a obtener reconocimiento como miembros del SNI; y (iv) poseer indicios de haber realizado comunicación científica, aunque no necesariamente de forma sistemática.

Se procedió a la identificación de los docentes que cumplieran con el perfil antes descrito, resultando en un grupo de 28, mismos que accedieron a participar y a quienes se localizó de forma directa. Se realizó una recolección de datos con estos docentes, a través de la metodología de entrevista a profundidad dirigida, utilizando los 34 indicadores de rendimiento de evaluación, utilizando también las evidencias de los últimos tres años de actividad científica de los 28 docentes. La entrevista a profundidad fue realizada de forma individual y en ámbitos cercanos a las actividades laborales del participante, donde fuera posible acceder a sus evidencias de comunicación científica.

En todos los casos, la recolección de datos se desarrolló de forma intermitente, tanto en el número de sesiones requeridas, como en el orden de los indicadores de rendimiento evaluados, según la disposición de tiempo del participante. Los datos recolectados permitieron clasificar a los indicadores de rendimiento en tres tipos: (i) los que se demuestran a través de evidencias tangibles, pero no a través de la demostración por acciones de ejecución demostrables (19 de 34 indicadores de rendimiento, agrupándose en: documentos científicos publicados, tesis dirigidas, materiales didácticos, proyectos, docencia, colaboración científica y acciones de divulgación científica); (ii) los que son producto de la autopercepción del participante (12 de 34 indicadores de rendimiento evaluados, caracterizados por: conocimiento de una lengua extranjera, redacción científica, uso de manuales de estilo, acceso a bases de datos y estrategias de búsqueda, derechos de autor, estadística, etc.); y (iii) los que son derivación a partir de las evidencias presentadas y consulta de fuentes alternas (3 de 34 indicadores de rendimiento evaluados, referidos a: citaciones, niveles de obsolescencia y nivel de producción científica sistemática). 
Una vez recolectados los datos y con fines de objetivarlos, se asignó a cada criterio de evaluación un puntaje, siendo el más alto de cada criterio el que se valore con 10 puntos y aplicando proporciones porcentuales al resto de los resultados bajo la siguiente fórmula:

$$
\mathrm{Vc}=\frac{(\mathrm{Nv} * \mathrm{Na})}{V m} \quad \mathrm{~V} \%=\frac{(\mathrm{Pv} * 100)}{P a}
$$

$\mathrm{Na}=$ número más alto

$\mathrm{Vm}=$ valor máximo

$\mathrm{Vc}=$ valor constante

$\mathrm{Nv}=$ número variante

$\mathrm{Pv}=$ puntaje variante $\sum \mathrm{Vc}$

$\mathrm{Pa}=$ puntaje más alto $=340$

$\mathrm{V} \%=$ valor porcentual
Considerado por un valor de 10 puntos para cada criterio.

Es el valor máximo de cada criterio del total de las encuestas.

Es el valor del número variante que se le dio al criterio multiplicado por el puntaje del número más alto entre el valor máximo.

El número variante es el valor asignado a cada criterio.

Suma de todos los Vc de cada una de las encuestas.

Se refiere al número de indicadores de rendimiento (34) multiplicado por el valor del número más alto (10).

Valor del puntaje total por encuesta representado en porcentaje.

Los resultados generales obtenidos en este grupo de investigación de 28 docentes con potencialidad científica son presentados primero de forma general por participante y de forma específica por criterio de evaluación (ver Anexo 2). Al ser la puntuación máxima posible de 340, se observó como puntaje mínimo 133 y 261 como máximo, con una media aritmética de 189,2 puntos, siento evidente la identificación de condiciones clave para ser consideradas dentro de los procesos de mejora (ver Figura 1).

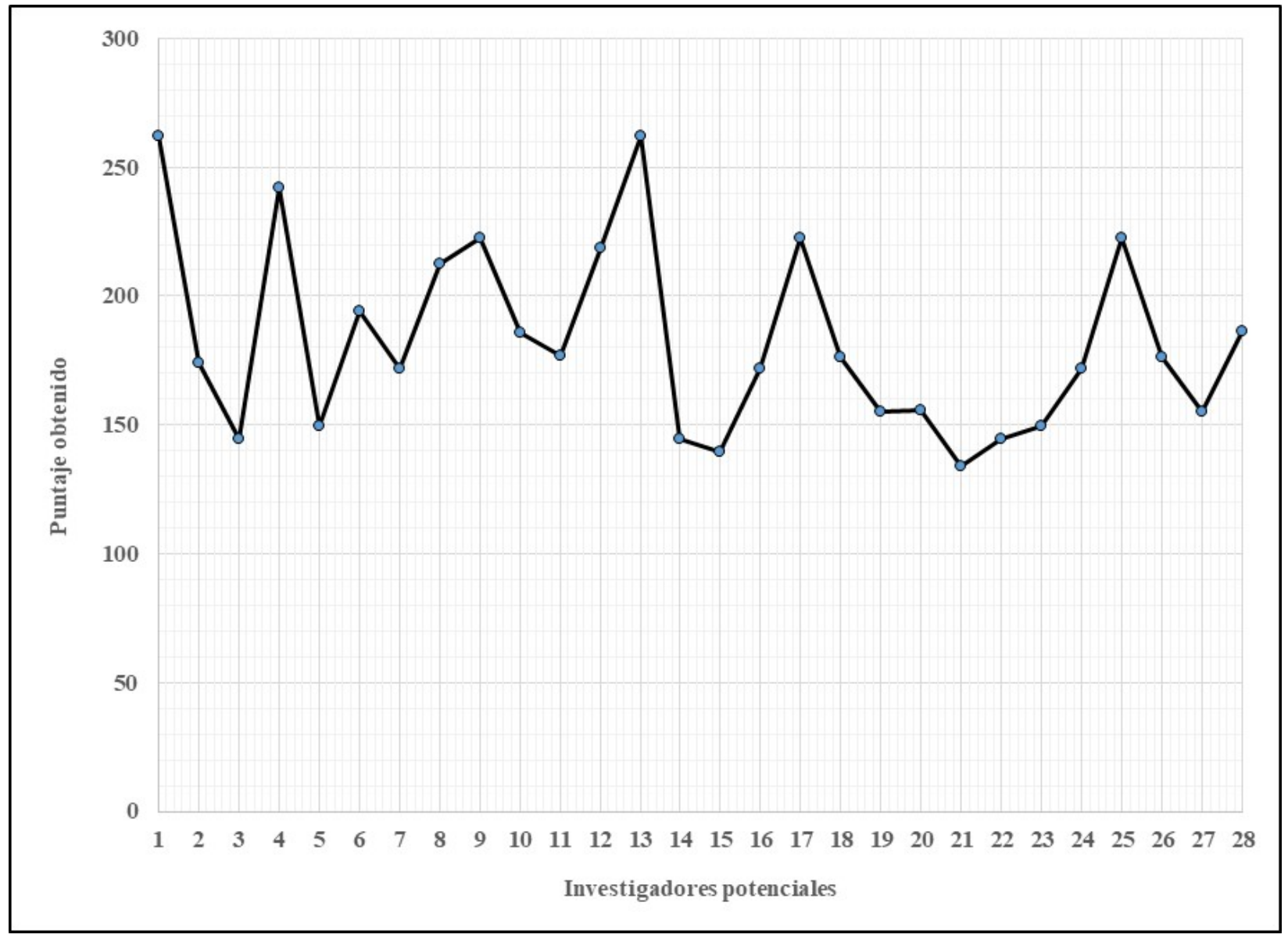

Figura 1. Valores totales por investigador potencial (Pv). 
Los valores totales (Pv) fueron convertidos a valores porcentuales (V\%), siendo la media aritmética de 53,81\% de rendimiento global y observándose que 17 investigadores potenciales (60,71\%) están por debajo de dicha media y solo 11 están por encima de la misma (39,28\%), siendo el porcentaje más alto 77,03\% y el más bajo 39,38\% (ver Figura 2).

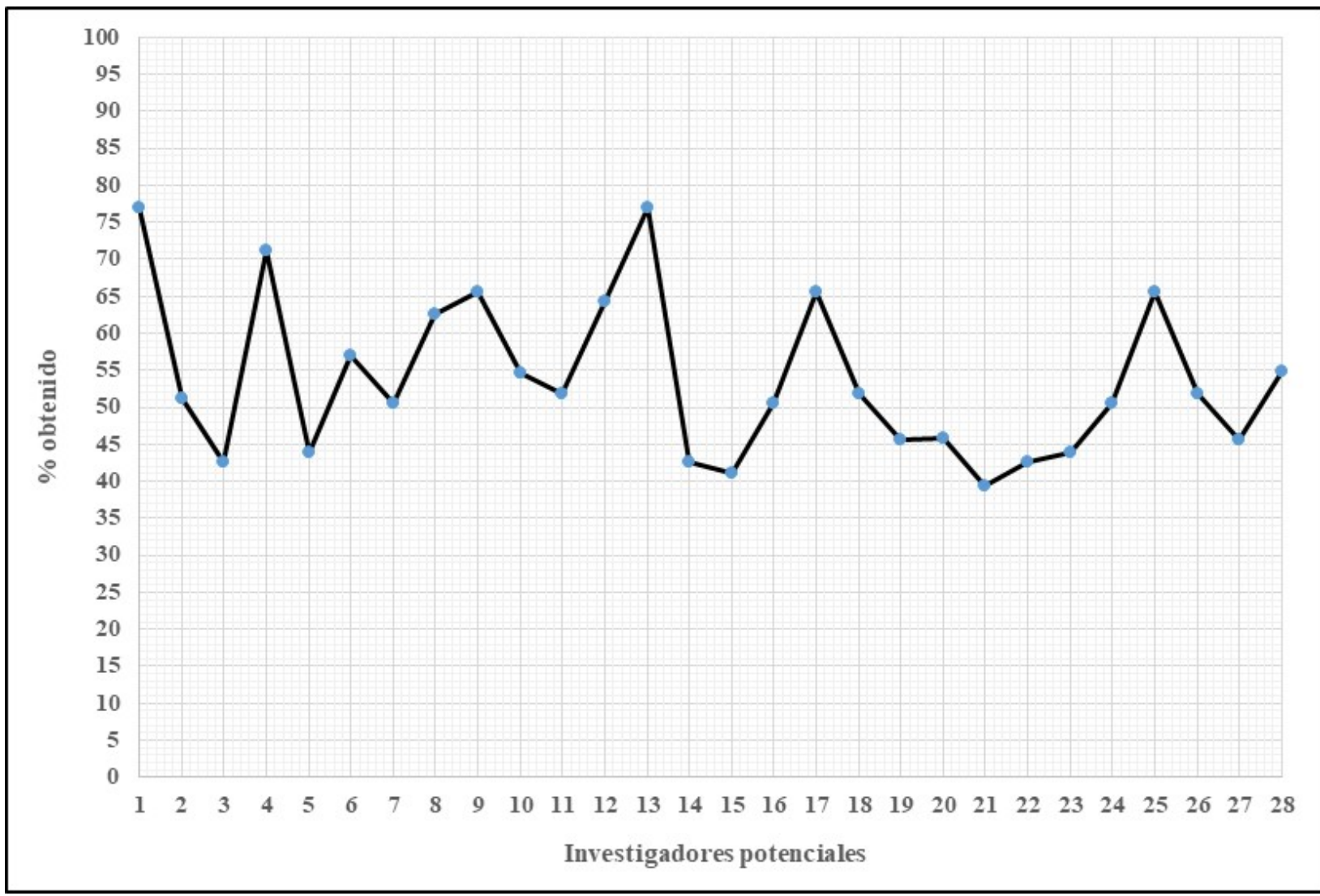

Figura 2. Valores porcentuales por investigador potencial (V\%).

La aplicación del conjunto de estándares demostró la posibilidad de llegar a resultados concretos con respecto a las condiciones observadas de forma individual y colectiva, lo cual es útil para la toma de decisiones sobre iniciativas de capacitación, incentivos y desarrollo institucional e individual. De los 34 indicadores de rendimiento evaluados, pueden ofrecerse las siguientes aseveraciones: (i) salvo en cuatro indicadores de rendimiento, en los que hubo un resultado positivo y unánime, en el resto existe alta variabilidad de resultados y, por lo tanto, se identificaron diversas debilidades en la competencia de comunicación científica de investigadores inmersos en el estudio (los resultados específicos por dimensión y criterio se incluyen en el Anexo 2); (ii) los resultados de aplicación deberán servir para la toma de decisiones en cuanto a aspectos de capacitación, programas de incentivos y probabilidades de identificar oportunidades de desarrollo; y (iii) los resultados de la productividad científica están determinados por la presencia o ausencia que se observe en las prioridades de las propias instituciones en las que se desempeña el investigador.

\section{CONCLUSIONES}

La comunicación científica y la ALFIN, como base de esta investigación, se convierten en un área de estudio de marcado impacto social que va aparejado a las nuevas condiciones y entornos informativos, tecnológicos y educacionales; especialmente en niveles superiores, donde se considera al docente como investigador involucrado en múltiples actividades. Actualmente, se valora el trabajo del docente e investigador a través del conocimiento que produce, de su utilidad práctica e incluso se pretende llegar a la demostración de que sus resultados de investigación llegan a impactar positivamente en la economía y la sociedad en general.

Los resultados de esta investigación responden a la necesidad de establecer estándares para consolidar los diversos indicadores de rendimiento que utilizan las entidades encargadas de medir la comunicación científica de las instituciones de educación superior e investigación, tanto para medir el desempeño en relación a la calidad científica 
de investigadores reconocidos como tales, así como para la incorporación de investigadores jóvenes. En ambos casos se exige la definición precisa de indicadores de rendimiento de evaluación debidamente normalizados, con los cuales se conozca de forma precisa los parámetros de medición que favorezcan el desarrollo de habilidades hacia la demostración de su desarrollo competitivo en comunicación científica.

Aunque se presenta un conjunto de estándares producto de un proceso de investigación, la propuesta no es definitiva, ya que pudieran sugerirse renglones alternativos y formas de valoración diferenciados al análisis hecho en esta investigación, a partir de su aplicación en investigadores potenciales. Debe tomarse en cuenta que la identificación de indicadores de rendimiento en las diferentes fases del estudio responde en gran medida a la forma en que el docente-investigador supone que va a ser evaluado por diferentes instancias, no tanto por lo que podría considerar como su compromiso social, valorando solo aspectos que lo posicionan dentro de sus propias instituciones; por ejemplo, en ningún momento se valoró la divulgación científica, las contribuciones a la apropiación social del conocimiento o a la generación de ciencia ciudadana.

Algunas consideraciones a futuro son las siguientes: (i) generar indicadores de rendimiento específicos para la transferencia de conocimiento e influir en el desarrollo del sector productivo a través de procesos de innovación o proyectos productivos, propiciando un estrecho vínculo con los sectores social y privado; (ii) contemplar criterios relacionados con la capacidad de gestión en la organización de eventos científicos (congresos, simposios o talleres); (iii) definir dentro de la dimensión de calidad académica y habilitación profesional, el criterio de ser invitado como árbitro de artículos científicos, proyectos y evaluación en pares; (iv) reforzar los aspectos éticos y legales, evitando la simulación al incluir autores sin participación real; (v) en el caso particular de las ciencias exactas, se recomienda agregar aspectos relacionados con la bioética y tomar en cuenta protocolos de bioseguridad; (vi) el empleo de estándares puede ser fundamental para la identificación de nuevos procesos de contratación y búsqueda de potencialidades; y (vii) prestar especial atención al elemento de las actitudes de los investigadores, al propiciar mayor participación voluntaria y comprometida.

\section{BIBLIOGRAFÍA}

AGUILAR JUSTO, M.O.; GALLEGOS RAMÍREZ, J.L. y MEDINA RAMÍREZ, R. Instrumento para evaluación de cuerpos académicos. Revista iberoamericana para la investigación y el desarrollo educativo, 2013, vol. 10, [en línea]. Disponible en: <http://ride.org.mx/1-11/index.php/RIDESECUNDARIO/article/viewFile/203/198> [Consulta: 14 de abril de 2019]

ANCHONDO-GRANADOS, R. Evaluación de competencias en comunicación científica: definición de un conjunto de estándares para docentes universitarios. Tesis de doctorado en Educación (Universidad Autónoma de Chihuahua, México, 2017) [en línea]. Disponible en: <repositorio.uach.mx/142/1/TESIS ROCÍO ANCHONDO GRANADOS, 2017.pdf> [Consulta: 14 de abril de 2019]

AREA MOREIRA, M. Educar para la sociedad informacional: Hacia el multialfabetismo. Revista portuguesa de pedagogía, 2008, vol. 42, nº 3, p. 7-22.

BUELA-CASAL, G. et al. Productividad y eficiencia en investigación por comunidades autónomas españolas según su financiación (2012). Revista iberoamericana de psicología y salud, 2015, vol. 6, n 1, p. 1-10.

BUELA-CASAL, A.; QUEVEDO-BLASCO, R. y GUILLÉN-RIQUELME, A. Ranking 2013 de investigación de las universidades públicas españolas. Psicothema, 2015, vol. 27, no 4, p. 317-326. DOI: 10.7334/psicothema2015.140.

CAAMAÑO, A. Física y química: complementos de formación disciplinar. Madrid, España: Grao, 2011.

CALZADA PRADO, J. y MARZAL, M.A. Incorporating Data Literacy into Information Literacy Programs: Core Competencies and Contents. Libri, 2013, vol. 63, n 2, p. 123-134.

CAÑEDO ANDALIA, R. Aproximaciones al impacto de la investigación en salud procedente de Cuba desde la perspectiva de Scopus. Correo científico médico, 2013, vol. 17, nº 3, p. 407-415.

CÁRDENAS CASIQUE, D.Y. Alfabetización científica: comprensión, producción y difusión de textos especializados con estudiantes de Comunicación Social. Ponencia presentada en el IV Congreso Venezolano de Investigadores de la Comunicación, 2013.

COLORADO ZÚÑIGA, M.D.P. y RODRÍGUEZ MOSQUERA, A.I. Proyecto de Alfabetización Científica y Tecnológica: una propuesta e implementación en la enseñanza de las ciencias naturales para la educación básica primaria. (Disertación doctoral, Universidad del Valle, Santiago de Cali, Colombia, 2014).

CONSEJO NACIONAL DE CIENCIA Y TECNOLOGÍA (a). Criterios SNI [en línea]. Disponible en: $<$ https://www.conacyt.gob.mx/index.php/sni/convocatorias-conacyt/convocatorias-sistema-nacional-deinvestigadores-sni/marco-legal-sni/criterios-sni> [Consulta: 14 de abril de 2019] 
CONSEJO NACIONAL DE CIENCIA Y TECNOLOGÍA (b). Criterios Específicos de Evaluación [en línea]. Disponible en: <https://www.conacyt.gob.mx/index.php/el-conacyt/sistema-nacional-de-investigadores/marcolegal/criterios-sni/13717-criterios-especificos-aiv/file> [Consulta: 14 de abril de 2019]

CONSEJO NACIONAL DE CIENCIA Y TECNOLOGÍA (c). Sistema de Centros de Investigación [en línea]. Disponible en: <https:/www.conacyt.gob.mx/index.php/el-conacyt/sistema-de-centros-de-investigacion> [Consulta: 14 de abril de 2019]

CONSEJO NACIONAL DE CIENCIA Y TECNOLOGÍA (d). Manual de Organización del Consejo Nacional de $\begin{array}{lllll}\text { Ciencia } & \text { y } & \text { Tecnología } & \text { [en } & \text { línea]. }\end{array}$ $<$ https://www.conacyt.gob.mx/cibiogem/images/cibiogem/normatividad/vigente/Manual-OrganizacionCONACYT.pdf $>$ [Consulta: 14 de abril de 2019]

DE ANDREA GONZÁLEZ, A. y TENA GODOY, F. La divulgación de conceptos fisicoquímicos a través de textos literarios ingleses, alemanes y españoles. En: PINTO CAÑÓN, G., (Coord). Enseñanza y divulgación de la química y la física. Madrid, España: Ibergaceta, 2012, p. 41-48.

DE PABLOS PONS, J. Universidad y sociedad del conocimiento: Las competencias informacionales y digitales. Universities and knowledge society journal, 2010, vol. 7, $\mathrm{n}^{\circ}$ 2, p. 6-16.

FONG, L. Comunicación de la ciencia e inclusión social. Desde la biblioteca, 2013, vol. 46, p. 43-53.

GARCÍA GÓMEZ, J. y MARTÍNEZ BERNAT, F.J. Cómo y qué enseñar de la biodiversidad en la alfabetización científica. Enseñanza de las ciencias, 2010, vol. 28, nº 2, p. 175-184.

GAVIDIA, V. Los retos de la divulgación y enseñanza científica en el próximo futuro. Didáctica de las ciencias experimentales y sociales, 2013, vol. 19, p. 91-102.

GONZÁLEZ ROSARIO, H. y HERNÁNDEZ LÁREZ, J.H. Aproximación a los elementos para potenciar la educación científica y la educación ambiental: Reflexiones desde la praxis. Revista de investigación, 2009, vol. 68, $n^{\circ} 33$, p. 251-274.

HARLEN, W. Evaluar la alfabetización científica en el programa de la OECD para la evaluación internacional de estudiantes (PISA). Enseñanza de las ciencias, 2002, vol. 20, n² 2, p. 209-216.

HERNÁNDEZ, S.A.; FLAVIA, C. y ZACCONI, M. Alfabetización científica. Química al alcance de todos. Ponencia presentada en el Congreso Iberoamericano de Educación. Universidad Nacional del Sur. Buenos Aires, Argentina, 2010.

IZQUIERDO, R. Normas para la evaluación de la producción científica de investigadores del programa 1042Documento 1042. Montevideo Uruguay: Universidad ORT Uruguay, 2013.

JIMÉNEZ, V. y OTERO, J. Acceso y procesamiento de información sobre problemas científicos con relevancia social: limitaciones en la alfabetización científica de los ciudadanos. Revista iberoamericana de ciencia, tecnología y sociedad, 2012, vol. 20, $\mathrm{n}^{\circ}$ 7, p. 29-54.

LARRAÍN SUTIL, A. El rol de la argumentación en la alfabetización científica. Estudios públicos, 2009, vol. 116, nº 4, p. 167-193.

LÓPEZ BLANCO, A. Relaciones entre la educación científica y la divulgación de la ciencia. Revista eureka sobre enseñanza y divulgación de las ciencias, 2004, vol. 1, nº 2, p. 70-86.

MARTÍN DÍAZ, M.J. Alfabetización científica: formación inicial, experiencia docente y pensamiento del profesorado. Enseñanza de las ciencias, 2005, Número extra, VII congreso, p. 1-4.

MARTÍN GUTIÉRREZ, A. y TYNER-AUSTIN, K. Educación para los medios, alfabetización mediática y competencia digital. Revista comunicar, 2012, vol. 19, n 38, p. 31-39.

ORTEGA, M.L. Cultura científica y educación. Revista educación y ciencia, 2011, vol. 7, nº 27, p. 53-62.

PALOMARES RUIZ, B.E.; SORDIA SALINAS, C. y DIMAS RANGEL, M.I. Indicadores que determinan el perfil deseable de los profesores en una dependencia de educación superior. Ponencia presentada en el Segundo Congreso Internacional de Investigación Educativa RIE-UANL, 2015.

PERALTA GONZÁLEZ, M.; SOLÍS CABRERA, F. y PERALTA SUÁREZ, L.M. Visibilidad e impacto de la producción científica de la Universidad Central "Marta Abreu" de Las Villas durante el período 2000-2008. Acimed, 2011, vol. 22, $\mathrm{n}^{\mathrm{o}}$ 1, p. 60-78.

SANZ MERINO, N. y LÓPEZ CEREZO, J.A. Cultura científica para la educación del siglo XXI. Revista iberoamericana de educación, Monográfico, 2012, vol. 58, p. 35-60.

SECRETARÍA DE EDUCACIÓN PÚBLICA. Lineamientos 2018 del Programa de Carrera Docente en UPES U040 (Fondo Extraordinario) [en línea]. Recuperado de: <https://www.ses.sep.gob.mx/pdfs/upes_u040_2018.pdf> [Consulta: 14 de abril de 2019]

SECRETARÍA DE EDUCACIÓN PÚBLICA (a). Reglas de Operación del Programa para el Desarrollo Profesional Docente para el Ejercicio Fiscal 2019 [en línea]. Disponible en: <http://www.dgesu.ses.sep.gob.mx/Documentos/DSA\%20gobmx/ROP2019.pdf> [Consulta: 14 de abril de 2019]

SECRETARÍA DE EDUCACIÓN PÚBLICA (b). Cuerpo Académico: conceptos básicos [en línea]. Disponible en: $<$ https://promep.sep.gob.mx/ca1/Conceptos2.html> [Consulta: 14 de abril de 2019] 
TARANGO, J. y MENDOZA GUILLÉN, G. Didáctica básica para la alfabetización informacional. Buenos Aires: Alfagrama, 2012.

TARANGO, J. y MACHIN-MASTROMATTEO, J.D. The Role of Information Professionals in the Knowledge Economy Skills, Profile and a Model for Supporting Scientific Production and Communication. Oxford, England: Elsevier-Chandos, 2017.

TEIXIDÓ MANS, C. Divulgar o vulgarizar: el problema del lenguaje. En: PINTO CAÑÓN, G. y MARTÍN SÁNCHEZ, M., editores. Enseñanza y divulgación de la química y la física. Madrid, España: Grupo Editorial Garceta, 2012, p. 17-24.

TÓJAR, J.C. y SERRANO, J. Ética e investigación educativa [en línea]. RELIEVE, 2012, vol. 6, nº 2. Recuperado de: <https://www.uv.es/relieve/v6n2/RELIEVEv6n2_2.htm> [Consulta: 14 de abril de 2019]

TORRES MESÍAS, A.; MORA GUERRERO, E.; GARZÓN VELÁZQUEZ, F. y CEBALLOS BORINA, N.E. Desarrollo de competencias científicas a través de la aplicación de estrategias didácticas alternativas: un enfoque a través de la enseñanza de las ciencias naturales. Tendencias, 2013, vol. XIV, nº 1, p. 87-215.

UNIVERSIDAD NACIONAL AUTÓNOMA DE MÉXICO - UNAM, Dirección General de Evaluación Institucional. Estudio comparativo de universidades mexicanas (ECUM) [en línea]. Recuperado de: $<$ http://www.dgei.unam.mx/hwp/ecum/> [Consulta: 14 de abril de 2019]

URIBE TIRADO, A. Interrelaciones entre veinte definiciones-descripciones del concepto de alfabetización en información: propuesta de macro-definición. Acimed, 2009, vol. 20, n 4, p. 1-22.

VALDÉS CUERVO, A.A.; VERA NORIEGA, J.A. y CARLOS MARTÍNEZ, E.A. Competencias científicas en estudiantes de posgrado de ciencias naturales en ingenierías. Sinéctica, $\mathrm{n}^{\circ} 39$ [en línea]. Recuperado de $<$ http://www.sinectica.iteso.mx/index.php?cur=39\&art=39_02> [Consulta: 14 de abril de 2019]

VILLARRUEL-FUENTES, M. Divulgar ciencia: compromiso social del investigador. Trilogía: Ciencia, Tecnología y Sociedad, 2013, vol. 8, p. 67-76.

WILSON, C. et al. Alfabetización mediática e informacional: currículum para profesores. Quito, Ecuador: UNESCO, 2011. 


\section{ANEXO 1. ESTÁNDARES SOBRE APTITUDES-FACULTADES EN COMUNICACIÓN CIENTÍFICA: INDICADORES DE RENDIMIENTO}

Estándar 1. Generación de conocimiento. El docente es capaz de diseñar y aplicar proyectos de investigación, además de mostrar habilidades de expresar las ideas de forma clara, concisa y coherente para llevar a cabo presentación de resultados, ya sea de forma oral o escrita, determinando su nivel de generación de conocimiento científico.

Indicadores de rendimiento: el docente es capaz de:

1. Publicar artículos en revistas especializadas en su área (nacionales e internacionales), indizadas, con factor de impacto suficiente para puntualizar la importancia de la actividad científica.

2. Publicar libros de autoría y de su disciplina científica en editoriales de prestigio (nacionales e internacionales).

3. Elaborar capítulos de libros en publicaciones monográficas en editoriales de calidad, lo cual le permita la colaboración con otros investigadores reconocidos.

4. Dirigir tesis, especialmente de nivel de posgrado y de su disciplina científica.

5. Producir material didáctico, estructurado en condiciones editoriales adecuadas.

6. Publicar artículos arbitrados en revistas especializadas, nacionales e internacionales.

7. Publicar memorias en extenso, con arbitraje, en eventos académicos nacionales e internacionales de calidad.

Estándar 2. Innovación en la generación de proyectos de investigación. El docente es capaz de aplicar procesos de innovación al generar proyectos de aplicación de conocimiento y desarrolla modelos y prototipos de procesos y productos de utilidad para su disciplina científica.

Indicadores de rendimiento: El docente es capaz de:

8. Elaborar y dirigir proyectos de generación y aplicación de conocimiento, con financiamiento interno de su propia institución de trabajo.

9. Elaborar y dirigir proyectos de generación y aplicación de conocimiento, con financiamiento externo, a través de fondos públicos y privados.

10. Elaborar y dirigir proyectos de generación y aplicación de conocimiento sin financiamiento.

11. Diseñar modelos y prototipos de procesos y productos particulares de su disciplina científica de estudio.

Estándar 3. Impacto en la producción científica. El docente es capaz de generar impacto a través de sus procesos de comunicación científica al recibir citas de sus publicaciones, tanto a través de productos individuales y en colaboración.

Indicadores de rendimiento: El docente es capaz de:

12. Recibir citas de sus publicaciones en revistas de prestigio internacional a través de los principales índices (Scopus y Web of Science).

13. Generar publicaciones científicas, individuales y en colaboración interna y externa.

14. Generar publicaciones con niveles de obsolescencia aceptables, según las propias exigencias de su disciplina científica.

Estándar 4. Calidad académica y habilitación profesional. El docente demuestra habilitación a través de la adquisición de grados académicos suficientes para desempeñarse como investigador, desarrollando actividades de comunicación científica, tanto en su lengua madre como en una distinta, lo cual le permita el acceso, traducción e interpretación de contenidos y por consecuencia, logre generar acciones en la divulgación y comunicación de contenidos científicos, tanto a través de publicaciones, como de la docencia en niveles de educación superior.

Indicadores de rendimiento: el docente es capaz de:

15. Poseer un grado académico suficiente (doctorado) que lo habilite para el ejercicio de la investigación y comunicación científica.

16. Impartir docencia en distintos niveles académicos superiores (licenciatura, maestría, doctorado y posdoctorado).

17. Demostrar conocimiento suficiente de una lengua extranjera que le permita acceder a traducir y difundir conocimientos científicos.

Estándar 5. Desarrollo en ámbitos para el ejercicio profesional. El docente es capaz de desarrollar actividades de investigación en sectores sociales y productivos, así como desempeñarse en programas académicos superiores acreditados, participar en la integración de redes nacionales e internacionales, dirigir grupos de investigación y estar inmerso en cuerpos colegiados especializados.

Indicadores de rendimiento: El docente es capaz de: 
18. Desempeñarse eficientemente sus labores de docente e investigador en programas académicos acreditados, de nivel superior, contribuyendo a su reconocimiento o nivel de prestigio o para fortalecerlos.

19. Desarrollar actividades de investigación que propicien la vinculación con los sectores sociales y productivos.

20. Participar en la integración de redes nacionales e internacionales, a través de las cuales es posible colaborar con profesionales de su disciplina científica.

21. Dirigir grupos de investigación y estar inmerso en cuerpos colegiados para el desarrollo de procesos de producción y comunicación científica.

Estándar 6. Reconocimiento de los procesos de investigación. El docente demuestra competencia de alfabetización informacional, digital y científica al ser capaz de reconocer los procesos de investigación.

Indicadores de rendimiento: el docente es capaz de:

22. Identificar y diferenciar los tipos de investigación, además de demostrar habilidad práctica para utilizar en sus investigaciones diseños experimentales y no experimentales.

23. Reconocer los procesos y tipos de investigación pertinentes para cada situación que demande desarrollar.

24. Aplicar los principios en redacción científica, manejar adecuadamente la estructura de referencias de la información científica y apegarse a manuales de estilo específicos.

25. Utilizar bases de datos y estrategias de búsqueda de información científica, demostrando capacidad de análisis y síntesis de contenidos relevantes y válidos.

26. Poseer conocimientos suficientes para identificar y usar documentos científicos de calidad, aplicando técnicas para evaluar la información.

27. Usar adecuadamente métodos estadísticos para el análisis de datos (estadística descriptiva e inferencial) que le permitan interpretar y generalizar resultados.

28. Manejar Tecnologías de la Información y Comunicación (TIC) para el desarrollo de sus procesos de producción y comunicación científica (redacción de documentos, administración del conocimiento, o software para el procesamiento de datos).

29. Poseer conocimientos suficientes para el desarrollo de protocolos y proyectos de investigación.

30. Identificar medios de comunicación científica, usar la literatura científica para comunicar y difundir resultados en medios de calidad, diferenciando el público al que van dirigidos, así como a los niveles de interpretación y generalización de resultados.

Estándar 7. Aspectos éticos y legales de la comunicación científica. El docente demuestra competencia en la aplicación correcta de los aspectos éticos y legales, además de comprender las problemáticas y cuestiones económicas, legales y sociales que se relacionan con el uso de la propiedad intelectual y los derechos de autor, además de ser capaz de acceder y utilizar información científica de forma ética y legal.

Indicadores de rendimiento: El docente es capaz de:

31. Aplicar las normas de derechos de autor, evitar caer en plagio y autoplagio.

32. Demostrar la capacidad para valorar aspectos económicos, tecnológicos y ecológicos de las consecuencias de su trabajo científico.

Estándar 8. Transferencia de conocimiento. El docente demuestra competencia a través de la participación en ponencias y ponencias magistrales para la comunicación y divulgación de la ciencia.

Indicadores de rendimiento: El docente es capaz de:

33. Participar como ponente magistral en ponencias, conferencias y seminarios especializados en eventos académicos nacionales e internacionales, reconocidos por su calidad.

34. Participar como conferencista en congresos nacionales e internaciones de alta calidad. 


\section{ANEXO 2. DESCRIPCIÓN DE LOS RESULTADOS DE LA APLICACIÓN DE LOS ESTÁNDARES PARA EVALUAR EL DESEMPEÑO DEL GRUPO DE DOCENTES PARTICIPANTES}

\begin{tabular}{|c|c|c|}
\hline Dimensión & \multirow{2}{*}{\begin{tabular}{ll} 
& \multicolumn{3}{|l|}{ Indicadores de rendimiento } \\
1. & Publicación de r artículos \\
& científicos con factor de impacto.
\end{tabular}} & \multirow{2}{*}{$\begin{array}{l}\text { Descripción del resultado } \\
3,57 \% \text { publica; } 10,71 \% \text { publican dentro de la } \\
\text { media aritmética; } 85,71 \% \text { han publicado al menos } \\
\text { un producto. }\end{array}$} \\
\hline \multirow[t]{7}{*}{$\begin{array}{ll}1 . & \text { Generación de } \\
\text { conocimiento. }\end{array}$} & & \\
\hline & $\begin{array}{l}\text { 2. Publicación de libros de autoría, } \\
\text { especializados y en editoriales de } \\
\text { prestigio. }\end{array}$ & $\begin{array}{l}28,57 \% \text { cumple y } 71,42 \% \text { no cumple (nula } \\
\text { participación). }\end{array}$ \\
\hline & $\begin{array}{l}\text { 3. Publicación de capítulos de libro } \\
\text { en colaboración con } \\
\text { investigadores reconocidos. }\end{array}$ & $\begin{array}{l}14,28 \% \text { participan } \\
\text { participación media; } 72,43 \% \text { participación nula. }\end{array}$ \\
\hline & 4. $\quad$ Dirección de tesis de posgrado. & $\begin{array}{l}3,57 \% \text { cumple; } 25 \% \text { no participa; } 71,43 \% \\
\text { participa. }\end{array}$ \\
\hline & 5. $\quad$ Producción de material didáctico. & $\begin{array}{l}21,42 \% \text { no tiene contribuciones; } 78,57 \% \text { sí lo } \\
\text { tiene; } 14,28 \% \text { está por encima de la media } \\
\text { aritmética; } 64,28 \% \text { está por debajo de la media } \\
\text { aritmética. }\end{array}$ \\
\hline & $\begin{array}{llr}\text { 6. } & \text { Publicación de artículos } \\
\text { científicos en revistas arbitradas. }\end{array}$ & $\begin{array}{l}\text { 31,57\% no tiene publicaciones; } 64,28 \% \text { tiene un } \\
\text { artículo arbitrado publicado. }\end{array}$ \\
\hline & $\begin{array}{ll}\text { 7. Publicación de memorias en } \\
\text { extenso con arbitraje. }\end{array}$ & $\begin{array}{llll}25 \% \text { no muestra evidencias; } & 75 \% & \text { muestra } \\
\text { evidencias. } & & & \\
\end{array}$ \\
\hline $\begin{array}{lrr}\text { 2. Innovación } & \text { en } & \text { la } \\
\text { generación } & & \text { de } \\
\text { proyectos } & \text { de }\end{array}$ & $\begin{array}{llrl}\text { 8. } & \begin{array}{l}\text { Elaboración y } \\
\text { proyectos con } \\
\text { interno. }\end{array} & \text { dirección de } \\
\text { financiamiento }\end{array}$ & $\begin{array}{l}53,57 \% \text { no muestran evidencia; } 43,43 \% \text { muestran } \\
\text { evidencia, de los cuales, el } 32,14 \% \text { están por } \\
\text { encima de la media aritmética. }\end{array}$ \\
\hline gación. & $\begin{array}{llr}\text { 9. } & \text { Elaboración y dirección de } \\
\text { proyectos con financiamiento } \\
\text { externo (fondos públicos y } \\
\text { privados). }\end{array}$ & $\begin{array}{l}50 \% \text { no ha participado; } 21,42 \% \text { está por encima } \\
\text { de la media aritmética; } 28,57 \% \text { por debajo de } \\
\text { esta. }\end{array}$ \\
\hline & $\begin{array}{l}\text { 10. Dirección de proyectos en } \\
\text { general. }\end{array}$ & 85,71\% no participa; $14,29 \%$ participa. \\
\hline & $\begin{array}{l}\text { 11. Desarrollo de modelos } \\
\text { prototipos. }\end{array}$ & $\begin{array}{l}\text { 7,14\% desarrolla modelos y prototipos; } 92,85 \% \\
\text { no lo hace. }\end{array}$ \\
\hline $\begin{array}{l}\text { 3. Impacto de la } \\
\text { producción científica. }\end{array}$ & 12. Recibir citaciones. & $\begin{array}{l}35,71 \% \text { tiene al menos una cita; } 64,28 \% \text { no tiene; } \\
3,57 \% \text { más de } 500 \text { citas. }\end{array}$ \\
\hline & $\begin{array}{l}\text { 13. Publicaciones colaborativas con } \\
\text { otros investigadores e } \\
\text { instituciones. }\end{array}$ & $\begin{array}{l}\text { El 39,28\% no poseen publicaciones individuales y } \\
\text { colectivas con otros autores e instituciones; el } \\
60,71 \% \text { si lo tiene, de los cuales, el } 29,41 \% \text { están } \\
\text { por encima de la media y el } 70,58 \% \text { están por } \\
\text { debajo de la media. }\end{array}$ \\
\hline & $\begin{array}{l}\text { 14. Niveles adecuados de } \\
\text { obsolescencia en la literatura } \\
\text { publicada. }\end{array}$ & 25\% no cumplen; $75 \%$ cumplen. \\
\hline $\begin{array}{l}\text { 4. Calidad académica y } \\
\text { habilitación }\end{array}$ & 15. Grado académico de doctorado. & $\begin{array}{l}71,42 \% \text { tienen grado de doctorado; } 28,57 \% \text { no lo } \\
\text { poseen. }\end{array}$ \\
\hline pr & $\begin{array}{l}\text { 16. Impartición de docencia en nivel } \\
\text { superior (licenciatura, maestría y } \\
\text { doctorado). }\end{array}$ & $\begin{array}{l}89,28 \% \text { imparte docencia a nivel superior; } \\
10,72 \% \text { no lo hace. }\end{array}$ \\
\hline & $\begin{array}{l}\text { 17. Dominio de una lengua extranjera } \\
\text { para acceso, traducción y } \\
\text { comunicación de contenidos } \\
\text { científicos. }\end{array}$ & $\begin{array}{l}\text { 10,71\% manifestó no ser competente; } 89,28 \% \text { si } \\
\text { es competente; } 3,57 \% \text { manejan dos lenguas } \\
\text { extranjeras. }\end{array}$ \\
\hline $\begin{array}{l}\text { 5. Ámbitos para el } \\
\text { ejercicio profesional. }\end{array}$ & $\begin{array}{l}\text { 18. Participación como miembro de } \\
\text { núcleos académicos básicos en } \\
\text { programas de posgrado } \\
\text { acreditados. }\end{array}$ & $100 \%$ \\
\hline & 19. Desarrollo de actividades de & $25 \%$ \\
\hline
\end{tabular}




\begin{tabular}{|c|c|c|}
\hline \multirow[t]{4}{*}{ Dimension } & Indicadores de rendimiento & Descripción del resultado \\
\hline & $\begin{array}{l}\text { investigación con } \\
\text { sociales y productivos. }\end{array}$ & \\
\hline & $\begin{array}{l}\text { 20. Participación en la integración de } \\
\text { redes nacionales e } \\
\text { internacionales. }\end{array}$ & 75\% no lo hace; 25\% pertenecen al menos a uno. \\
\hline & $\begin{array}{l}\text { 21. Dirección de grupos de } \\
\text { investigación } y \text { cuerpos } \\
\text { colegiados para el desarrollo de } \\
\text { producción científica. }\end{array}$ & 67,85\% dirige o participa; 32,14\% no participa. \\
\hline \multirow[t]{9}{*}{$\begin{array}{l}\text { 6. Reconocimiento } \\
\text { los procesos } \\
\text { investigación. }\end{array}$} & $\begin{array}{l}\text { 22. Identificación y diferenciación de } \\
\text { tipos de investigación, diseños } \\
\text { experimentales } \\
\text { experimentales. }\end{array}$ & $100 \%$ cumple. \\
\hline & $\begin{array}{l}\text { 23. Redacción científica, manejo } \\
\text { adecuado de referencias y apego } \\
\text { a manuales de estilo. }\end{array}$ & $\begin{array}{l}85,71 \% \text { manejan este criterio; } 14,28 \% \text { demandan } \\
\text { formación. }\end{array}$ \\
\hline & $\begin{array}{l}\text { 24. Conocimiento y uso frecuente de } \\
\text { bases de datos y estrategias de } \\
\text { búsqueda de información } \\
\text { científica. }\end{array}$ & $100 \%$ cumple. \\
\hline & $\begin{array}{l}\text { 25. Producción científica sistemática } \\
\text { y no aislada. }\end{array}$ & $\begin{array}{l}\text { 10,71\% no presenta evidencia; } 89,28 \% \text { presenta } \\
\text { evidencia; } 8 \% \text { muestra alta suficiencia; } 92 \% \\
\text { muestra baja evidencia. }\end{array}$ \\
\hline & 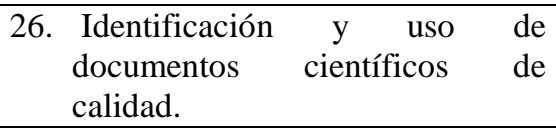 & 100\% cumple. \\
\hline & $\begin{array}{l}\text { 27. Uso adecuado de métodos } \\
\text { estadísticos (descriptivos e } \\
\text { inferenciales) para el análisis de } \\
\text { datos. }\end{array}$ & 71,42\% cumplen; 28,57\% no cumple. \\
\hline & $\begin{array}{l}\text { 28. Manejo de Tecnologías de la } \\
\text { Información } \\
\text { Comunicaciones } y \\
\text { (redacción de documentos, } \\
\text { software para procesamiento de } \\
\text { datos, etc.). }\end{array}$ & 100\% cumple. \\
\hline & $\begin{array}{l}\text { 29. Desarrollo de protocolos y } \\
\text { proyectos de investigación. }\end{array}$ & $100 \%$ cumple. \\
\hline & $\begin{array}{l}\text { 30. Identificación } \\
\begin{array}{l}\text { comunicación } \\
\text { científica. }\end{array}\end{array}$ & 100\% cumple. \\
\hline \multirow[t]{2}{*}{ 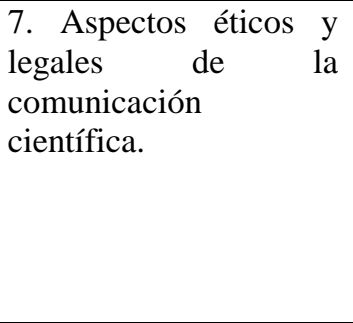 } & $\begin{array}{l}\text { 31. Conocimiento y aplicación de } \\
\text { normas de derechos de autor y } \\
\text { propiedad intelectual, evitando } \\
\text { plagio y autoplagio. }\end{array}$ & 100\% cumple. \\
\hline & $\begin{array}{l}\text { 32. Valoración de aspectos } \\
\text { económicos, tecnológicos y } \\
\text { ecológicos de las consecuencias } \\
\text { del trabajo científico. }\end{array}$ & 100\% cumple. \\
\hline \multirow[t]{2}{*}{$\begin{array}{l}\text { 8. Transferencia de } \\
\text { conocimiento. }\end{array}$} & $\begin{array}{l}\text { 33. Participación como ponente } \\
\text { magistral en eventos académicos } \\
\text { de prestigio. }\end{array}$ & 25\% participa; 75\% no participa. \\
\hline & $\begin{array}{l}\text { 34. Participación como ponente o } \\
\text { conferencista en eventos } \\
\text { académicos de prestigio. }\end{array}$ & $\begin{array}{l}64,28 \% \text { han participado como ponentes; } 35,71 \% \\
\text { no lo han hecho. }\end{array}$ \\
\hline
\end{tabular}

